\title{
Comparison of Prooxidant-antioxidant Balance between Patients with High Grade Gliomas (IV) and Control Group
}

\author{
Elnaz Farajirad ${ }^{1}$, Kazem Ghaemi ${ }^{2}$, Mehdi Khajavi ${ }^{3}$, Daryoush Hamidi Alamdari ${ }^{4}$, Mohammad Hassan \\ Arjmand $^{5}$, Mohammad Farajirad ${ }^{6 *}$ \\ ${ }^{1}$ Head of Ghaem Health Center, Mashhad University of Medical Sciences, Mashhad, Iran. \\ ${ }^{2}$ Associate Professor of Neurosurgery, Head of Neurosurgery Department, Faculty of Medicine, Birjand University of Medical Sciences, Birjand, \\ Iran. \\ ${ }^{3}$ Resident of Neurosurgery, Neurosurgery Department, Ghaem Hospital, School of Medicine, Mashhad University of Medical Sciences, Mashhad, \\ Iran. \\ ${ }^{4}$ Stem Cell and Regenerative Medicine Research Group, Biochemistry \& Nutrition Research Center, Faculty of Medicine, Mashhad University of \\ Medical Sciences, Mashhad, Iran. \\ ${ }^{5}$ Master of Biochemistry, Surgery Department, Ghaem Hospital, School of Medicine, Mashhad University of Medical Sciences, Mashhad, Iran. \\ ${ }^{6}$ Professor of Neurosurgery, Head of Neurosurgery Department, Faculty of Medicine, Mashhad University of Medical Sciences, Mashhad, Iran. \\ * Corresponding Author: Professor of Neurosurgery, Head of Neurosurgery Department, Faculty of medicine, Mashhad University of Medical \\ Sciences, Mashhad, Iran. Tel+985138424834, Fax:+9838425878 Email: Farajim@mums.ac.ir
}

Article Type: Research Article

Received: March 1, 2014, Last revised: September 15, 2015, Accepted: September 19, 2015

\section{Abstract}

Background \& Aim: The most common primary brain tumors of the central nervous system are gliomas. Among a number of different biomolecular events, a strong relation between oxidative stress pathways and the development of this cancer has been proved. Oxidative stress (OS) is the consequence of an imbalance between pro-oxidants and antioxidants towards pro-oxidants. The pro-oxidants cause lipid peroxidation of cell membranes, protein, DNA oxidation and changes in brain cell growth. In this study, the pro-oxidant-antioxidant balance (PAB) was determined in patients with gliomas.

Methods \& Materials/Patients: Sera of 49 patients with high grade glioma (grade IV WHO) which is called glioblastoma multiform (GBM) and 49 healthy subjects were collected and PAB test was measured.

Results: A significant increase of PAB value was observed in patients with GBM (158.10 $\pm 85.71 \mathrm{HK}$ unit) in comparison to control group $(74.54 \pm 33.54 \mathrm{HK})$.

Conclusion: The PAB assay showed the oxidative stress in glioblastoma. In further research, this easy elucidation of oxidative stress in these patients can be applied to evaluate the effectiveness of antioxidant therapies in patients with GBM.

Keywords: Prooxidant-antioxidant; Balance; High Grade Gliomas

Please cite this paper as: Farajirad E, Ghaemi K, Khajavi M, Hamidi Alamdari D, Arjmand M.H, Farajirad M. Comparison of Prooxidantantioxidant Balance between Patients with High Grade Gliomas (IV) and Control Group. Iran. J. Neurosurg. 2015;1(2):27-30.

\section{Introduction}

Oxidative stress is a consequence of imbalance between pro-oxidants and anti-oxidants in favor of pro-oxidants. Prooxidants (O2., H2O2, OH., etc.) derive either from metabolic processes or from external sources and can potentially react with the body's own molecules. There are different mechanisms for pro-oxidants actions including lipid peroxidation of the cellular membranes, oxidation of proteins and DNA resulting changes in chromosome structure, genetic mutation, modulation of cell growth and genomic instability and all of these mechanisms can play a role in carcinogenesis. On the other hand, human body has some defense mechanisms against pro-oxidants to neutralize their effects. Some components of this defense system are intrinsic enzymes like super oxide dismutase (SOD), glutathione peroxidase, catalase, uric acid and billirubin, while others are supplied from extrinsic sources such as vitamin C, betacarotene and vitamin E (1-2). Glioma grade IV (GBM) is the most common primary brain tumor with abnormal cells which have grown out of control and the expected survival for GBM is less than 12 months from the time of diagnosis (3). Prooxidants play a role in numerous diseases including diabetes mellitus, epilepsy, schizophrenia, cardiovascular diseases, neurological disorders, the aging process, cancers and trauma $(4,5)$. Considering the high consumption of oxygen in brain tissue compared to other organs-with brain consuming $20 \%$ of oxygen which enters human body- brain is very sensitive to oxidative stress $(6,7)$. Understanding how neural tissue changes its normal behavior is fundamental to developing effective therapies for human diseases. Various methods measure the total oxidants (such as TOS and FOX assays) or antioxidants (such as FRAP and ORAC assays), separately. However, the pitfall of these methods is that one cannot trust their results about the 
exact value of pro-oxidants and anti-oxidants because prooxidants and anti-oxidants have cross-reactions on each other, and this fact can produce unreliable results. Considering this matter, a new method which is called PAB assay (Pro-oxidantsAntioxidants Balance Assay) have been developed recently to measure the balance of oxidants and antioxidants simultaneously in one experiment and give a redox index which is measured by HK unit. The higher HK value means worse oxidative stress (8-9). In this study, we determined the prooxidant-antioxidant balance in patients with high grade glioma brain tumor (grade IV) by PAB assay. This method can reliably show how worse is oxidative stress in high grade glioma and the result of this study can be used in future studies for evaluating the therapeutic effects of antioxidants by prescribing them to GBM patients and measuring PAB assay in them thereafter. To the best of our knowledge, this is the first time that PAB is determined in patients with grade IV glioma.

\section{Methods \& Materials/Patients}

\section{Chemical agents for $P A B$ assay:}

TMB powder (3, 3', 5, 5'-Tetramethylbenzidine, Fluka), peroxidase enzyme (Applichem: 230 U/mg, A3791, 0005, Darmstadt, Germany), chloramine $\mathrm{T}$ trihydrate (Applichem: A4331, Darmstadt, Germany), hydrogen peroxide (30\%) (Merck). Molecular biology grade reagents were used and preparations were done in double distilled water.

\section{Subjects:}

Sera of 49 patients with high grade (IV) GMB and 49 healthy subjects as the control group were collected. Samples were collected during summer 2013. The PAB was measured. The study protocol was approved by the Ethics Committee for Clinical Research of the Mashhad University of Medical Sciences (MUMS), Iran. Blood samples of each subject were collected and then sent to the laboratory for serum separation. Samples were centrifuged at $1500 \mathrm{~g}$ for $15 \mathrm{~min}$ at room temperature to obtain serum and stored at $-80^{\circ} \mathrm{c}$. Hemolytic samples were excluded from analysis. Of the patients admitted to Neurosurgery ward of Qaem Hospital, Mashhad in summer 2013, 58 patients suspected to GBM according to their imaging and meeting all of our inclusion criteria including having primary GBM without any systemic disease, being non-smoker without tumor recurrence and without any history of previous chemoradiotherapy were enrolled. Their blood samples were then taken and sent for evaluating PAB. After operation, the diagnosis of grade IV glioma was confirmed histopathologically in 49 patients and then accordingly 49 healthy patients who were demographically similar to our cases were selected. Thereafter, PAB value results were reviewed and those 9 patients in whom GBM was not proved were excluded.

\section{Method OF PAB ASSAY:}

PAB assay is the only available test that can measure the balance of oxidants and antioxidants simultaneously in one experiment. It uses two different kinds of reactions: one is an enzymatic reaction where the chromogen TMB is oxidized to a color cation by peroxides and the second is a chemical reaction where the TMB cation is reduced to a colorless compound by antioxidants (10-11). The photometric absorbance is then compared with the absorbances given by a series of standard solutions that are made by mixing varying proportions $(0-$
$100 \%$ ) of hydrogen peroxide with uric acid (8). A low PAB value means that antioxidants are present at greater concentration than oxidants, while a high PAB value means more oxidants are present than antioxidants. The standard solutions were prepared by mixing varying proportions $(0-100 \%)$ of $250 \mathrm{mM}$ hydrogen peroxide with $3 \mathrm{mM}$ uric acid (in $10 \mathrm{mM} \mathrm{NaOH}$ ). The TMB powder $(60 \mathrm{mg})$ was dissolved in $10 \mathrm{ml}$ DMSO. For preparation of the TMB cation solution, $400 \mathrm{ml}$ of the TMB/ DMSO solution was added to $20 \mathrm{ml}$ of acetate buffer $(0.05 \mathrm{M}$ buffer, $\mathrm{pH} 4.5)$, and then $70 \mathrm{ml}$ of fresh chloramine $\mathrm{T}(100 \mathrm{mM})$ solution was added. The solution was mixed well and incubated for 2 hours at room temperature in a dark place and then $25 \mathrm{U}$ of peroxidase enzyme solution was added. This mixture was dispensed into $1 \mathrm{ml}$ aliquots and stored at $-20^{\circ} \mathrm{C}$. The TMB solution was prepared by adding $200 \mu \mathrm{TMB} / \mathrm{DMSO}$ to $10 \mathrm{ml}$ of acetate buffer (0.05 M buffer, pH 5.8) and the working solution was prepared by mixing $1 \mathrm{ml}$ TMB cation solution with $10 \mathrm{ml}$ TMB solution. This working solution was incubated for 2 min at room temperature in a dark place and immediately used. Ten microliters of each sample, standard, or blank (distilled water) were mixed with $200 \mu$ l of working solution in each well of a 96 well plate, which was then incubated in a dark place at $37^{\circ} \mathrm{C}$ for 12 mins. At the end of incubation, $100 \mu 1$ of $2 \mathrm{~N} \mathrm{HCl}$ was added to each well and the optical density (OD) was measured with an ELISA reader at $450 \mathrm{~nm}$, with a reference wavelength of 620 or $570 \mathrm{~nm}$. A standard curve was generated from the values of the standard samples. The values of the PAB assay are expressed in arbitrary units based on the percentage of hydrogen peroxide in the standard solution. The values of the unknown samples were then calculated based on the values obtained from the generated standard curve.

\section{Results}

In this study, SPSS software (Version 16) was used for statistical analysis. All parameters were given as mean \pm S.D. The group comparisons were assessed by the independent t-test. The significance level was considered less than 0.05 with a confidence interval of $95 \%$. PAB value of control group and case group were $74.54 \pm 33.54$ (HK unit) and $158.10 \pm 85.71$ (HK unit), respectively (Figure 1). HK is an arbitrary unit used by inventors of PAB method (Hamidi \& Koliakos) (8). There was a significant increase of oxidative stress in patients with GBM (Grade IV) in comparison to control group (P-value $<0.05$ ).

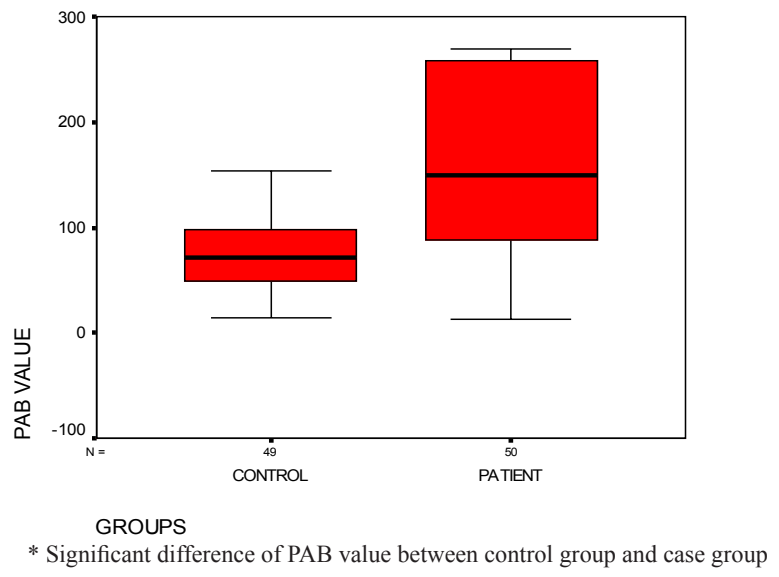

Figure 1. PAB Value in Control Group and Group with GMB Tumor (Mean and the standard error/standard deviation of PAB value) 


\section{Discussion}

This study showed the increased OS in patients with high grade (IV) glioma tumor in comparison to healthy group using PAB assay. The creation of glioma tumor is a complex process involved neoplastic changes of cells, resistance to apoptosis, lack of cell cycle control and angiogenesis (12). Many studies showed that OS has a substantial role in these processes and modulation of OS can have a therapeutic potential in treatment (13-14). It is demonstrated that pro-inflammatory cytokine and OS could interact and provoke each other and contribute to the development and progression of some diseases such as diabetes and cancer. OS can cause increasing cytokine production by many different mechanisms. The increased POX levels, acting similar second messengers, are well known to mediate inflammatory signaling by activating various protein kinases such as JNK, PI3K, PKC and PLC. These kinases could stimulate redox sensitive transcription factors such as STAT and NF-KB. The activation of transcription factors lead to the transcriptional activation of inflammatory cytokines (TNF $\alpha$, IL-1, IL-6, IL-8, and IL-18, etc.), chemokines (chemoattractant protein-1, etc.) and growth factors (transforming growth factor- $\beta$, monocyte, connective tissue growth factor, etc). NF-KB has a role in proliferation and survival of glioblastoma cell line. Some studies showed that activation of NF-KB factor protect some glioblastoma cells lines in vitro from chemotherapeutic agents. Data confirmed that tumor grade is associated to NF-KB activity and its activity increases progressively with malignancy (15). TGF- $\beta 2$ is produced by human GBM cell lines and involved in the regulation of both suppression of anti-tumor immune surveillance and angiogenesis in malignant gliomas (16). Malignant gliomas produce IL-10 as a local immunosuppression factor. Levels of expression are correlated with grade of tumor (1). Different studies showed that the marker of oxidative stress, PGE2, is produced in malignant gliomas and possibly contributes to the ability of the tumor cells to escape the immune surveillance (16). Cyclooxygenase as an enzymatic pro-oxidant, plays a key role in inflammation through the transformation of arachidonic acid to inflammatory molecules. High Cyclooxygenase staining predicts a poor prognosis in patients with glioma in general and in patients with glioblastoma multiform (WHO grade IV) in particular (17). Also, it is demonstrated that antioxidants can down-regulate the pro-inflammatory cytokines through two possible mechanisms: firstly, through their effect on transcription factors that are regulated by redox status, and secondly by influencing PGE2 synthesis. During prolonged oxidative stress, changes in brain antioxidant enzymes activities, including superoxide dismutase (SOD), glutathione peroxidase (GPX), and catalase (CAT), glutathione transferases (GTSs) appear. These enzymes normally act to prevent or decrease brain damages caused by free radicals in excess. However, they are controlled by polymorphic genes which can be altered by free radicals, leading to dysfunctions in enzymes' activity. Their role in brain tumors genesis is sustained by several studies. Rao (2000) showed that the red-blood-cell activity levels of SOD decreased for most types of intracranial neoplasm (18).

\section{Conclusion}

This study showed the ability of PAB assay in detection of OS in patients with high grade glioma (IV). According to literature review, the role of OS in development of brain tumors has been demonstrated. Now there is an urgent need to determine the OS and monitor the effect of antioxidant therapy in these patients.
PAB assay can provide this facility to overcome the shortage of method to easy elucidation of OS. It is suggested that in further research, this assay can be applied to develop the effective antioxidant therapies for devising strategies to lessen or delay the the progression and/or complications in glioma.

\section{Funding}

None.

\section{Conflicts of Interest}

The authors declare that they have no conflicts of interests.

\section{References}

1. Halliwell B. Oxidative stress and neurodegeneration: where are we now? J Neurochem. 2006; 97:1634-1658.

2. Alamdari DH, Ghayour-Mobarhan M, Tavallaie S, Parizadeh MR, Moohebati $\mathrm{M}$, Ghafoori F, et al. Prooxidant-antioxidant balance as a new risk factor in patients with angiographically defined coronary artery disease. ClinBiochem. 2007; 41:375-80.

3. Ashby, L.S.; Ryken, T.C. Management of malignant glioma: steady progress with multimodal approaches. Neurosurg. Focus 2006, 20, E3.

4. Bhatti P, Stewart PA, Hutchinson A, Rothman N, Linet MS, Inskip PD, et al Lead exposure, polymorphisms in genes related to oxidative stress, and risk of adult brain tumors. Cancer Epidemiol Biomarkers Prev. 2009 Jun;18(6):1841-8.

5. Virgolici B, Mohora M, Stoian I, Lixandru D, Gaman L, Paveliu F A comparative oxidative stress study-obesity with and without diabetes mellitus. Rom J Intern Med, Jan2005; 43(3-4) pg. 261-8.)

6. Somani SM. Exereise, drugs, and tissue specific antioxidant system. Pharmacology in sport and exercise 1996;57-95.

7. Evans PH. Free radicals in brain metabolism and pathology.Br Med Bull 1999;49:577-87. 2-

8. Alamdari DH, Paletas K, Pegiou T, Sarigianni M, Befani C, Koliakos G. Novel assay for the evaluation of the prooxidant-antioxidant balance, before and after antioxidant vitamin administration in type II diabetes patients. ClinBiochem 2007; 40:248-54

9. Erel O. A novel automated method to measure total antioxidant response against potent free radical reactions. ClinBiochem 2004; 37:112-119.

10. Erel O.A new automated colorimetric method for measuring total oxidant status. ClinBiochem 2005; 38:1103-11011.

11. Huettner, C.; Czub, S.; Kerkau, S.; Roggendorf, W.; Tonn, J.C. Interleukin 10 is expressed in human gliomas in vivo and increases glioma cell proliferation and motility in vitro. Anticancer Res. 1997, 17, 3217-3224.

12. Conti A, Gulì C, La Torre D, Tomasello C, Filippo F. Role of Inflammation and Oxidative Stress Mediators in Gliomas. Cancers 2010, 2, 693-712; doi:10.3390/cancers2020693

13. Schwartzbaum J, Cornwell D. Oxidant Stress and GlioblastomaMultiforme Risk: Serum Antioxidants, $\gamma$-GlutamylTranspeptidase, and Ferritin. Nutrition and Cancer 2000; 38(1): 40-49.

14. Tanriverdi T, Hanimoglu H, Kacira T, ZihniSanus G, Kemerdere R, Atukeren $\mathrm{P}$, et al. Glutathione peroxidase, glutathione reductase and protein oxidation in patients with glioblastomamultiforme and transitional meningioma. J Cancer Res and ClinOncol 2007; 133(9):627-633

15. Conti A, Guli C, La Torre D, Tomasello C, Angileri F., Aguennouz M H. Role of Inflammation and Oxidative Stress Mediators in Gliomas, Cancers 2010, (2) pg.693-712

16. Platten, M.; Wick, W; Weller, M. Malignant glioma biology: role for TGFbeta in growth,motility, angiogenesis, and immune escape. Microsc. Res. Tech. 2001, 52, 401-410.

17. Shono, T.; Tofilon, P.J.; Bruner, J.M.; Owolabi, O.; Lang, F.F. Cyclooxygenase-2 expression in human gliomas: prognostic significance and molecular correlations. Cancer Res. 2001, 61,4375-4381. 
18. Rao GM, Rao AV, Raja A, Rao S, Rao A. Role of antioxidant enzymes in brain tumours. ClinChimActa. 2000(296) pg.203-212

\section{Comments}

Farajirad and colleagues compared the pro-oxidant-antioxidant balance (PAB) in 49 patients with grade IV glioma and 49 healthy patients. They found the significant difference between these two groups. The results seem to be an addition to the literature and even to the science. However, there are some concerns. First, the detail of healthy control group is an important issue. How did they find healthy control patients? How did they ask healthy patients to receive a few cc of their blood? How did they obey ethical issues in both case and control groups? Did they sign informed consent for blood sampling? Did patients with glioma know that the blood sampling is not directly related to the management of their disease?

The second issue is about the time of receiving blood samples in cases and controls: Was it performed in the same time for cases and controls? Authors used PAB assay in their previous study for control group of patients with coronary artery disease (2). If they used similar healthy cases for both studies, they should mention it. If there is difference in PAB assay in control groups in this study and control group in the previous study, how they explain it?

The third issue is the detail of statistical tests and the version of SPSS used which should be written in the methods section not in the results section.

The fourth issue is the detail of sex, age, and other demographic variables in cases and controls which should be the first table of the results and would consider before publication.

The fifth issue is about the figure of the study. We need to have more detail of red area and for two linear bars for the box and whisker plot in text and in figure legend. Was the difference statistically significant? If yes, what was the exact $\mathrm{P}$ value? It is not enough to mention just less than 0.05 . What is the $95 \%$ confidence interval?

Finally, the above-mentioned points are helpful to promote our critical thinking and our research papers in future studies in Neurosurgery.

Vafa Rahimi-Movaghar, MD, Professor of Neurosurgery, Sina Trauma and Surgery Research Center, Tehran University of Medical Sciences, Tehran, Iran

\section{References}

1. Farajirad E, Ghaemi K, Khajavi M, Alamdari DH, Arjmand MH, Faraji rad M. Comparison of Prooxidant-antioxidant Balance between Patients with High Grade Gliomas (IV) and Control Group. IrJNS. 2015;1(2): 27-30.

2. Alamdari DH, Ghayour-Mobarhan M, Tavallaie S, Parizadeh MR, Moohebati M, Ghafoori F, et al. Prooxidant-antioxidant balance as a new risk factor in patients with angiographically defined coronary artery disease. ClinBiochem. 2007; 41:375-80. 